\title{
Probing Mid-Mantle Heterogeneity Using PKP Coda Waves
}

\author{
Michael A.H. Hedlin and Peter M. Shearer \\ Cecil H. and Ida M. Green Institute of Geophysics and Planetary Physics \\ Scripps Institution of Oceanography, University of California, San Diego
}

\begin{abstract}
We investigate the utility of $P K P$ coda waves for studying weak scattering from small-scale heterogeneity in the mid-mantle. Coda waves are potentially a useful probe of heterogeneity at shallow depths because this energy is not preferentially scattered near the CMB, as $P K P$ precursors are, but is due to scattering at all depths. $P K P$ coda waves have not been used for this purpose historically because of interference with other late-arriving energy due to near-surface resonance and scattering. We have analyzed 3624 recordings of $P K P$ precursors and coda made by stations in the IRIS Global Seismographic Network. To study the range and time dependence of the scattered waves, we binned and stacked envelopes of the recordings. $P K P$ scattered waves increase in amplitude rapidly with range as predicted by scattering theory. At ranges below $\sim 125^{\circ}$, we predict and observe essentially no scattered energy preceding $P K P$. Coda amplitudes at these ranges are independent of range and provide an estimate of energy due to near-surface effects we can expect at all ranges. We use the average coda amplitude at ranges from 120 to $125^{\circ}$ to correct coda amplitudes at other ranges. $P K P$ coda waves show a strong dependence on time and range and are clearly influenced by scattering in the lower mantle. PKP coda waves, however, do not provide a tighter constraint on the vertical distribution of mantle heterogeneity than is provided by precursors. This is due, in part, to relatively large scatter in coda amplitudes as revealed by a resampling analysis. Modeling shows that $P K P$ coda amplitudes are not highly sensitive to the vertical distribution of heterogeneity in the mantle. To illustrate this we consider single-scattering in two extreme models of mantle heterogeneity. One allows heterogeneity just at the CMB; the other includes heterogeneity throughout the mantle. The amplitudes of precursors are tightly constrained by our stack and support our earlier conclusion that small-scale heterogeneity is uniformly distributed throughout the lower mantle. The best-fit model includes $8 \mathrm{~km}$ scale length heterogeneity with an RMS velocity contrast throughout the mantle of $1 \%$.
\end{abstract}

\section{INTRODUCTION}

Since the pioneering work of Haddon and Cleary in the early 1970's, the high-frequency energy that precedes the inner core phase $P K P(d f)$ has been known to result from scattering in the mantle (Haddon, 1972; Haddon and Cleary, 1974; Figure 1). These scattered waves arrive free of interference from latearriving near-surface scattered waves and thus provide a unique window into the small-scale structure of the deep Earth. Numerous studies have used recordings of these precursors to investigate the physical properties of small-scale heterogeneity in the mantle (e.g. Doornbos 1972; Husebye et al., 1976; Hedlin et al., 1997). Although a recent analysis by Hedlin et al. (1997) used GSN recordings of the precursors to study heterogeneity throughout the lower mantle, the geometry of the precursors favors studies of the deepest mantle as the preponderance of early arrivals come from great depth. Hedlin et al. (1997) modeled the weak scattering that gives rise to precursors using the Born approximation and found that the gradual growth of the precursors with time and range favored a model that included heterogeneity distributed with equal strength at all depths in the lower mantle. Models that include heterogeneity just at the CMB or within D" produce early arrivals, and thus sharper onsets than are seen in the data. This surprising result awaits confirmation by an independent analysis, as models involving multiple scattering near the $\mathrm{CMB}$ might also be able to explain the $P K P$ precursor observations.

$P K P$ coda waves are due largely to near surface scattering and resonance, but also include contributions from scattering throughout the mantle. Evidence that lower mantle scattering contributes energy to $P K P$ coda can be found in raw data (Figure 2). Recorded $P K P$ coda amplitude variations with time and 

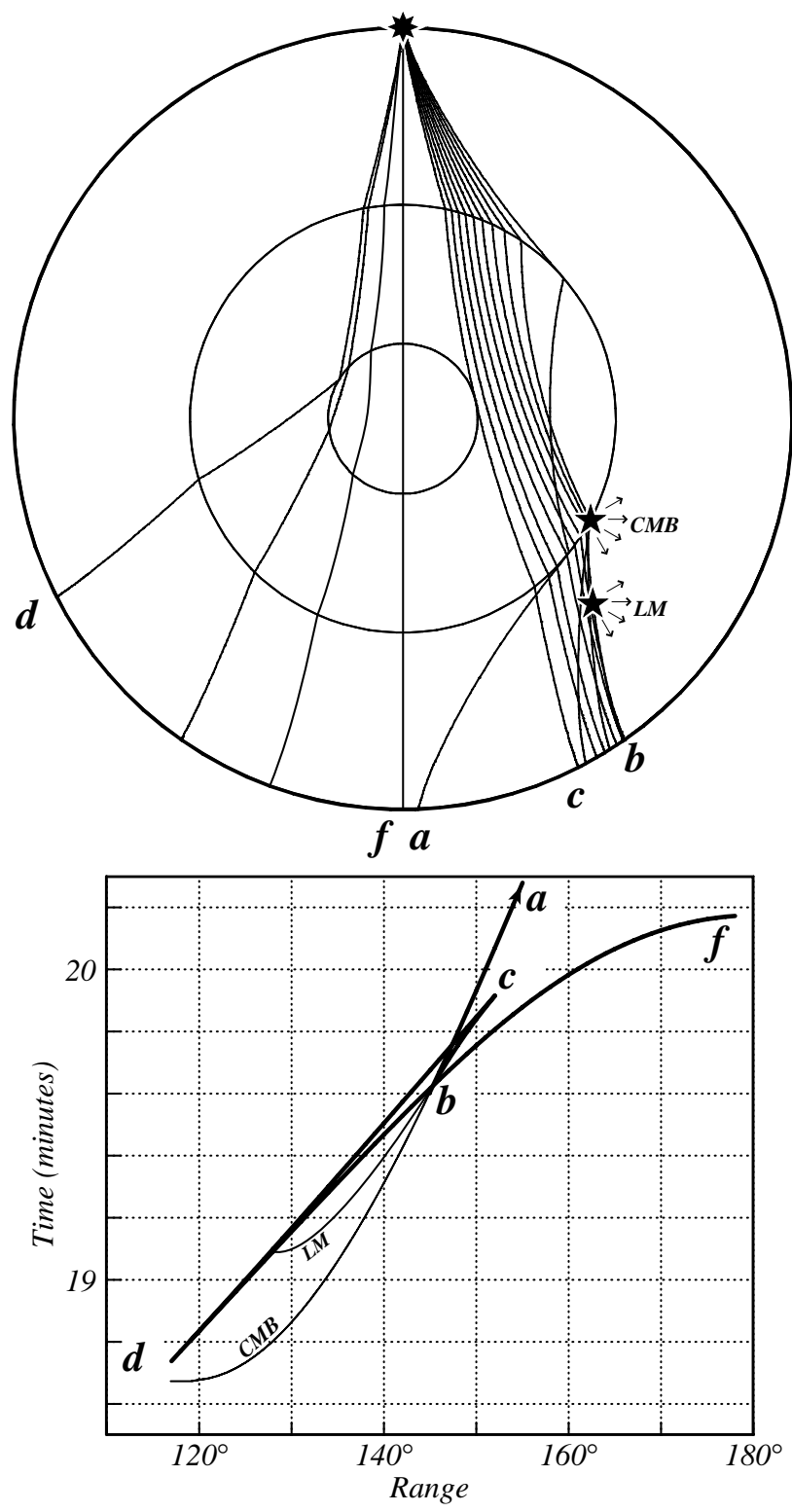

Figure 1. Ray paths and travel times of the different branches of $P K P$ for a source at the surface. The retrograde $a b$ and prograde $b c$ branches refract through the outer core. The $c d$ branch is reflected from the inner core boundary. The $d f$ branch refracts through the inner core. Scattering involving the $a b$ or $b c$ branch can produce precursory arrivals as well as later arrivals that contribute to the df coda. Scattering of $d f$ will not cause $d f$ precursors but will also contribute to the $d f$ coda. Two hypothetical scatterers (labeled CMB and LM) produce precursors that can arrive no earlier than the labeled curves in the lower panel. The deepest scatterers will give rise to the earliest precursors. Although we have depicted scattering from $P K P$ to $P$ near downward, adjustment of the amplitude to remove the receiver, precursors can also result from scat- pre-event noise. The binning procedure grouped tering from $P$ to $P K P$ on the source side. range mirror those observed in the precursors. This basic observation suggests that we might be able to put tighter constraints on the strength on heterogeneity in the mantle using coda waves provided that we are able to correctly compensate coda amplitudes for near surface effects. It is this idea that we test in this paper.

\section{DATA}

We have analyzed 3624 recordings of $P K P$ precursors and coda that were made between day 151 of 1988 to the end of 1999 by stations in the Incorporated Research Institutions for Seismology (IRIS) Global Seismographic Network (GSN). These recordings were selected from a much larger dataset on the basis of pre-event noise levels. We rejected traces that exhibited noise levels that were high or varied sharply with time. The events in the culled dataset occurred at depths from the nearsurface to $657 \mathrm{~km}$. Sixty percent of the events occurred within $100 \mathrm{~km}$ of the free-surface. The events were assigned body wave magnitudes between 5.0 and 7.3. Although the GSN stations are distributed across the globe, the tight range limits on the recordings $\left(120\right.$ to $\left.145^{\circ}\right)$ resulted in a rather uneven sampling of the Earth (Hedlin and Shearer, 2000). Some areas are not sampled at all.

\section{PRELIMINARY DATA ANALYSIS}

The traces in Figure 2 reveal a strong dependence of the precursors and coda waves on range and time. The precursors and coda increase in amplitude relative to $P K P$ from 120 to $144^{\circ}$. Energetic coda is seen in most traces to 60 seconds after the arrival of $P K P$. The traces, however, also reveal variations that are clearly not global in origin but are regional. One notable example of this is the trace at $\sim 137^{\circ}$ which is dominated by $P K P$. This trace implies weak scattering within the small volume of the mantle it samples. An earlier paper (Hedlin and Shearer, 2000) examined regional variations in scattering. This paper is concerned with global characteristics of the data. To constrain global average scattering strength, we binned each recording into the appropriate $1^{\circ}$ bin, after correcting for event depth. Each trace was filtered between 0.7 to $2.5 \mathrm{~Hz}$ and converted to an envelope. Prior to stacking, we applied a small, recordings simply on the basis of recording range 


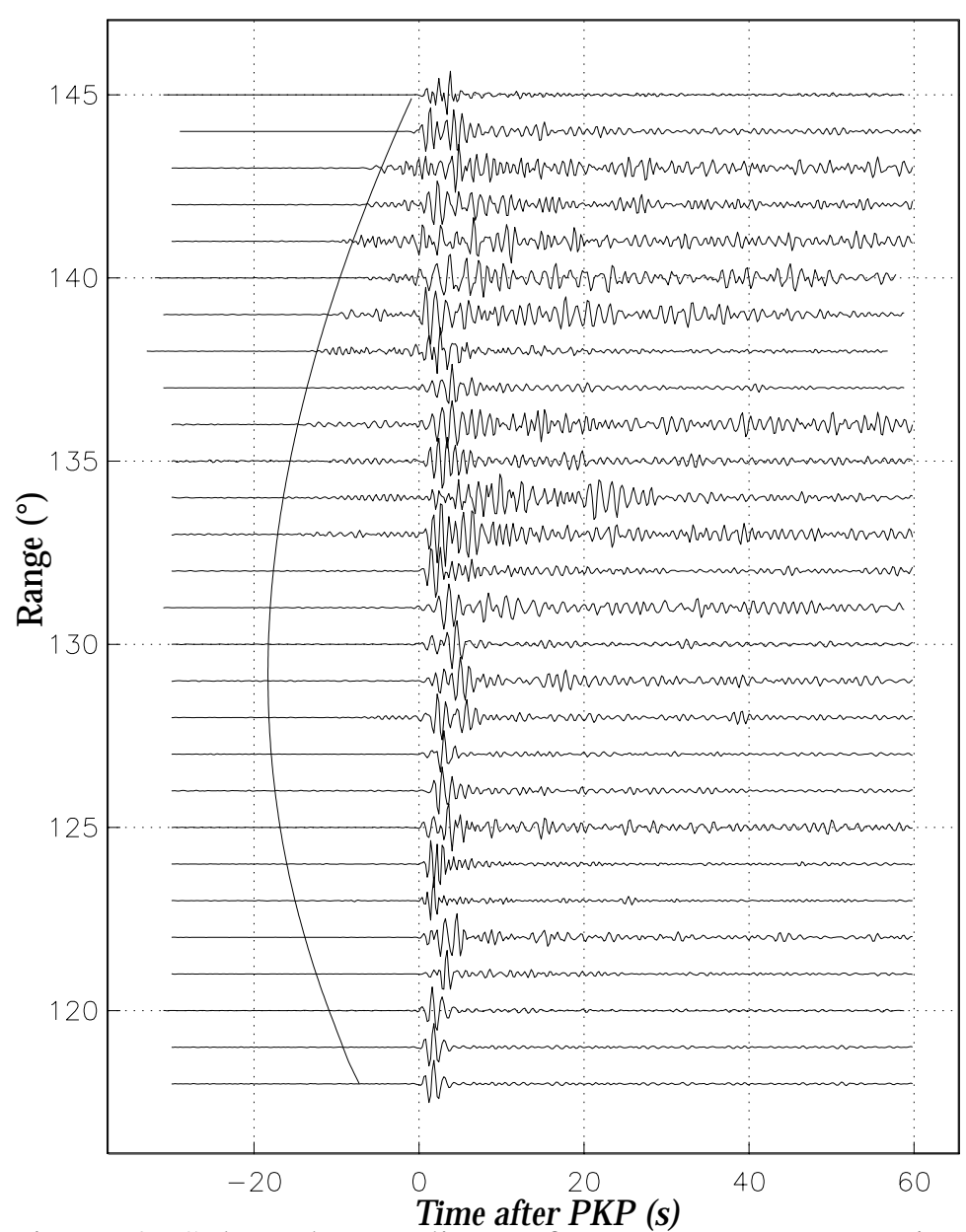

Figure 2. Selected recordings of $P K P$ precursors, main phase and coda made by stations in the Incorporated Institutions for Seismology (IRIS) Global Seismographic Network (GSN) between 118 and $145^{\circ}$. The data have been bandpassed between 0.7 and $2.5 \mathrm{~Hz}$ and are aligned on the $P K P(d f)$ onset. The traces reveal a gradual increase in the amplitude of the precursors and coda waves as a function of range. The curve indicates the minimum travel time for energy single-scattered at the CMB. and not on the location of either the source or the receiver. The stacked traces thus provide an image of the average characteristics of the arrivals.

The stacks shown in Figure 3 indicate that coda amplitudes at ranges from 120 to $125^{\circ}$ are nearly independent of range. No precursor energy is observed at these ranges. Between 125 and $144^{\circ}$ we observe a gradual increase in the amplitude of the precursors and coda. The precursors at these ranges increase in amplitude from the onset to $P K P$. After $P K P$, the coda amplitudes decay gradually with time. Scattering simulations (Hedlin et al., 1997) indicate that most of the energy at ranges below $125^{\circ}$ is due to near surface effects. To illustrate the amount by which the amplitude of the precursors and coda increases from the low levels seen at ranges below $145^{\circ}$ we plotted a copy of the average stack from 120 to $125^{\circ}$ at all ranges (see the dashed traces). This range dependence is unlikely due to near surface effects but we believe arises from scattering within the mantle. At $145^{\circ}$, the traces are dominated by $P K P$ as the $b$ caustic arrives at this range. All scattered waves are small in amplitude relative to $P K P$. The suites of curves in Figure 3 indicate the minimum travel time for single-scattered energy scattered at depths ranging from the CMB to $2200 \mathrm{~km}$ above. This figure illustrates that most scattered waves will follow $P K P$

\section{THE DEPTH EXTENT OF MANTLE SCATTERING}

Hedlin et al. (1997) considered the factors listed in the previous section and modeled a stack of 1600 $P K P$ precursor recordings using Rayleigh-Born scattering theory (Chernov, 1960) applied to the exponential autocorrelation function. The modeling indicated that the global average small-scale heterogeneity has a scale length of $\sim 8 \mathrm{~km}$ and is distributed uniformly throughout the lower-mantle with an RMS velocity contrast of $1 \%$.

We are attempting to re-examine the vertical distribution of heterogeneity in the mantle using coda waves to check the earlier study. This requires careful consideration of the paths energy might take through the core before or after scattering in the mantle. Propagation through the mantle from the surface to the CMB involves no triplications. Propagation from the CMB back to the surface after propagation through the core is along one of four branches of $P K P$ (Figure 1). The earliest arriving branch at ranges between $\sim 119$ and $132^{\circ}$ is $b c$ which represents prograde refraction through the outer core. The $a b$ branch lags behind by a few seconds at most. The $a b$ and $b c$ branches alone can yield precursors to PKP. These branches were considered by Hedlin et al. (1997). The other branches can be 


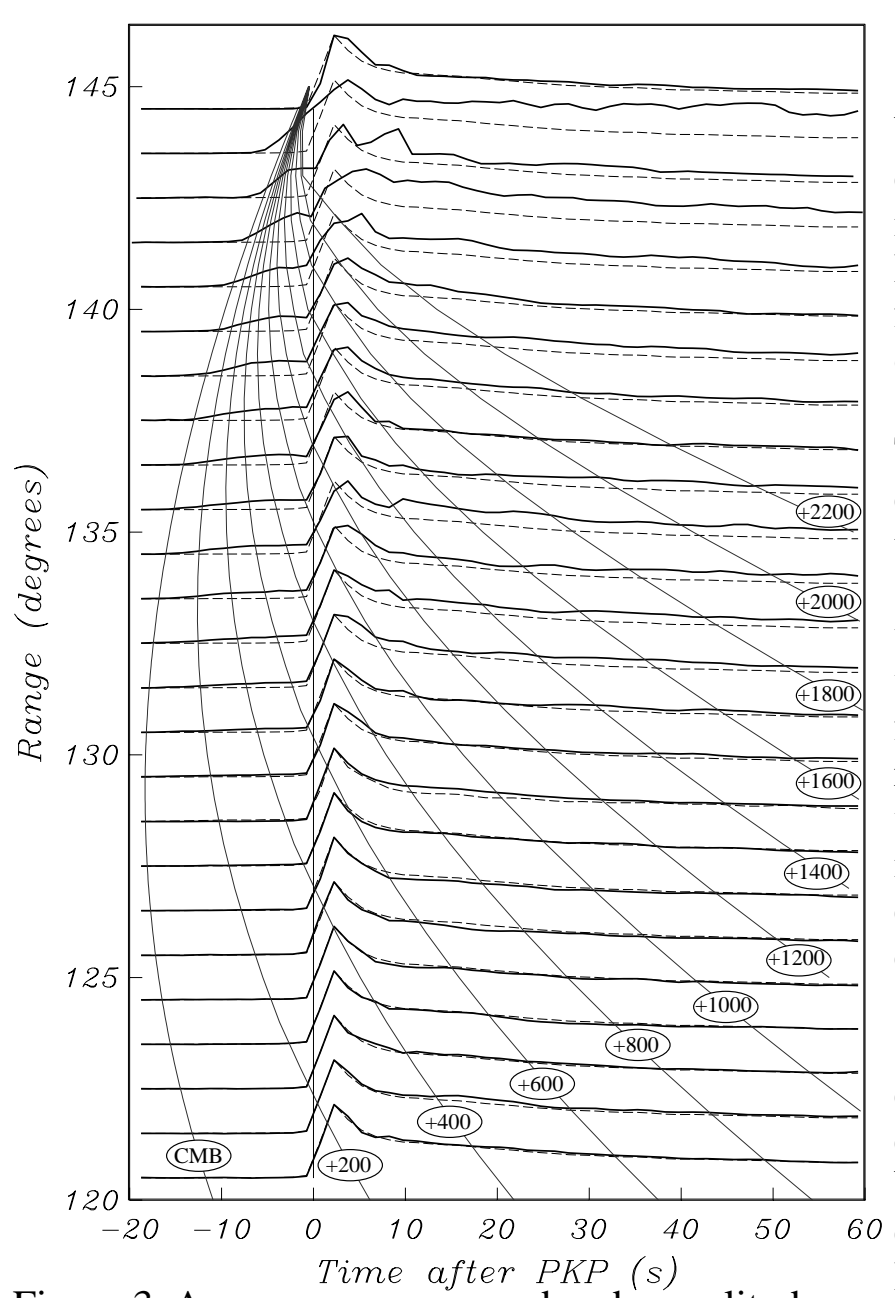

Figure 3. Average precursor and coda amplitudes as a function of time and source-receiver range as obtained from a waveform stack of 3624 seismograms. Times are relative to the onset of $P K P(d f)$. Each stacked trace is normalized to the peak $P K P(d f)$ amplitude (at about 2 seconds). The theoretical onset time from the PREM velocity model for single-scattering at a range of depths from the $\mathrm{CMB}$ to $2200 \mathrm{~km}$ above the CMB are shown as the curved lines. Note the increase in precursor and coda amplitudes with range and the gradual decay of coda amplitudes with time. The dashed curve at each range represents the average stack between 120 and $125^{\circ}$.

averaging of coda amplitudes removes regional variations and produces an estimate of the average near-surface energy levels we can expect at all ranges. The range independent average also includes energy that results from scattering in the mantle and propagation through the inner core along the $d f$ branch. Our simulations indicate that the $d f$ branch has a weak dependence on range when compared with the $a b$ and $b c$ branches which are strongly influenced by the $b$ caustic. The simulations also clearly show that regardless of what scattering model is used, the $a b$ and $b c$ branches produce essentially no scattered energy before or after $P K P$ at ranges below $125^{\circ}$. To take into account, and remove from consideration, near-surface effects and the inner core refracted scattered energy, we subtract the average coda amplitude observed at ranges below $125^{\circ}$ from all bins. The average stacked coda from 120 to $125^{\circ}$ possesses minor variations with time. To ensure that this structure is not introduced into the stacks by the correction procedure, we approximate the average stack using a standard coda decay 


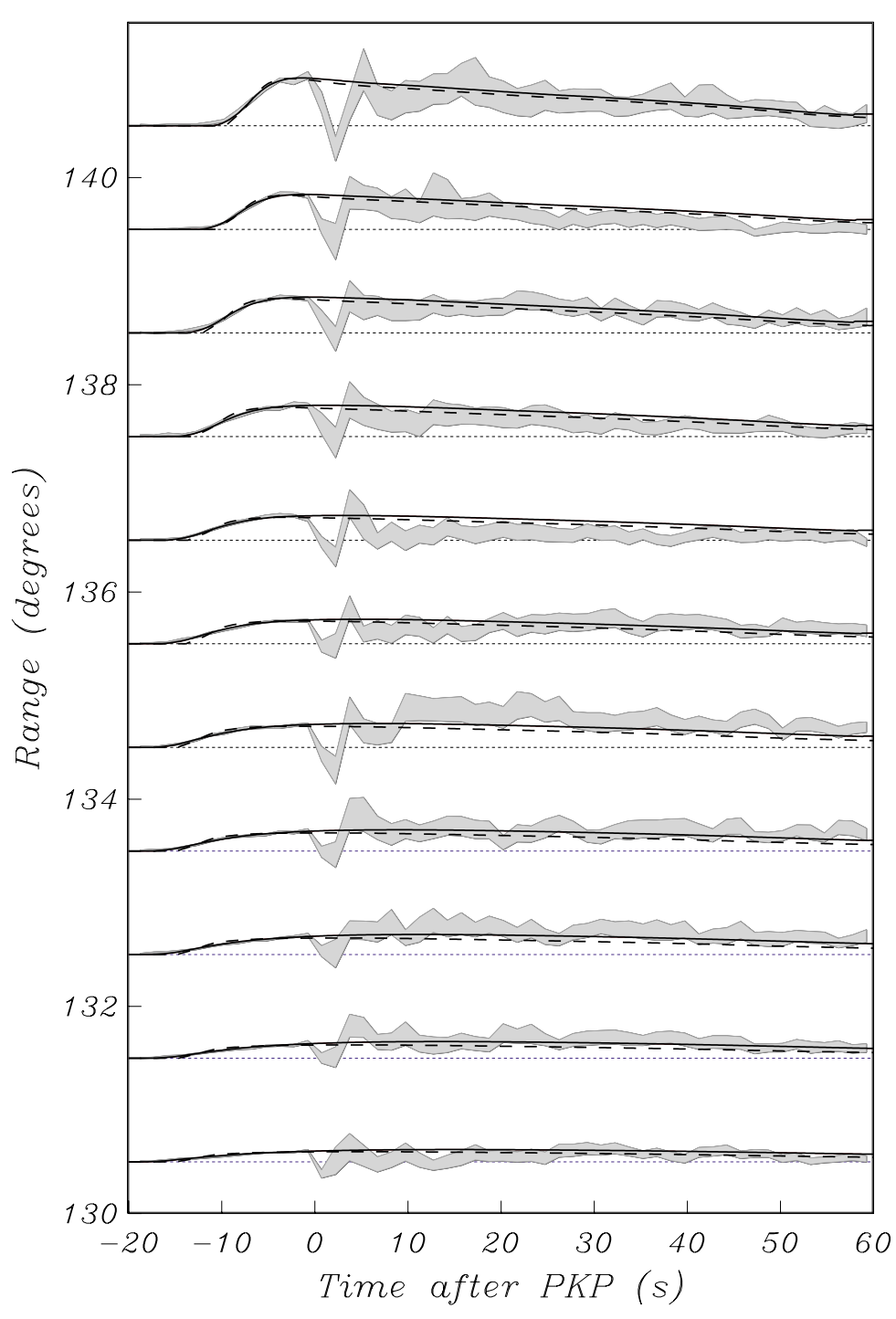

Figure 4. Observed $P K P(d f)$ precursor and adjusted coda amplitudes compared to theoretical predictions of two models of mantle heterogeneity. The whole-mantle simulations are shown by the solid curve, the CMB only simulations are represented by the dashed curves. The data and their standard errors are indicated by the grey shaded regions. Coda decay is explained equally well by these two extreme models of mantle heterogeneity. model (Pechmann et al., 2000). We assume the coda envelope amplitude as a function of time, $\mathrm{A}(\mathrm{t})$, can be described as follows:

$$
\log [\mathrm{A}(\mathrm{t})]=\log [\text { Ao }]-\alpha \log \left(\mathrm{t}-\mathrm{t}_{\mathrm{p}}\right),
$$

where $\mathrm{t}_{\mathrm{p}}$ is the time of the $P K P$ onset, Ao is the onset amplitude, $\alpha$ is a free fitting parameter. We deduct the best fit exponential decay function from the bins at all ranges. The correction applies only to the data points at times after the onset of $P K P$.

The result is given in Figure 4. We have shown the bins between 130 and $141^{\circ}$. The bins at greater ranges are difficult to interpret due to proximity to the $b$ caustic. The bins at ranges below $130^{\circ}$ provide little insight as there is very little energy before $P K P$ or after $P K P$ once the correction for $d f$ and the near-surface effects is made. At each range we show the stacked, and corrected, data and the standard errors from a resampling analysis (Efron and Tibshirani, 1991). The standard errors increase after the arrival of $P K P$. This is expected as coda amplitudes are largely due to near surface effects. These effects depend on the depth of the event. Most events were located within $100 \mathrm{~km}$ of the surface. Some events were $>600$ $\mathrm{km}$ in depth. While we have observed robust range independent estimates of coda amplitudes at ranges below $125^{\circ}$, and have inferred similar behavior at greater ranges, wide variability in the amplitudes that are present in individual traces is revealed by the resampling analysis.

Despite the scatter in the corrected PKP coda amplitudes shown in Figure 4, it is clear that the coda energy remains well above zero even after corrections for near-surface scattering are applied. This implies that scattering from the $\mathrm{CMB}$ and/or deep mantle is a significant contributor to $P K P$ coda energy at these ranges. This result could have been anticipated from Figure 3, given the observed increase in $P K P$ coda amplitude for those ranges where such deep scattering could occur. One goal of this paper is to test whether these $P K P$ coda observations can place new constraints on the depth extent of lower mantle scattering.

Predicted results from two different models are shown in Figure 4. One model (represented by the dashed curves) allows 8-km scale length heterogeneity within a 20-km-thick mantle layer immediately 
above the $\mathrm{CMB}$ and models the effect of scattering at or very close to the CMB (short-wavelength topography on the CMB would produce similar effects). The second model (solid curves) allows 8-km heterogeneity to be uniformly distributed throughout the mantle. For each model, we adjusted the RMS amplitude of the velocity variations to obtain the best fit to the PKP precursors. Our simulations reveal that coda decay rates are nearly independent of the vertical distribution of heterogeneity in the mantle. The CMB-only model predicts slightly more rapid decay of coda amplitudes after $P K P$. This difference is seen at all ranges. Taking into account uncertainty about the coda amplitudes as determined from a resampling analysis, our stacks indicate that both models are in nearly equal agreement with the data. Both predict an increase in energy before $P K P$ and then a gradual decay.

However, as noted in Hedlin et al. (1997), the two models do not produce equal fits to the PKP precursors. The model that includes heterogeneity just at the $\mathrm{CMB}$ produces onsets that are more rapid than observed. In addition, the $\mathrm{CMB}$ scattering model requires much larger velocity perturbations to achieve the same amplitude of scattering. The whole mantle model requires RMS velocity perturbations between 1 and $2 \%$ whereas the CMB scattering model requires perturbations of 5.5 to $7.5 \%$ within the deepest $20 \mathrm{~km}$ of the mantle.

\section{CONCLUSIONS}

Our analysis of 3624 teleseismic recordings of $P K P$ precursors and coda indicates that coda arrivals do not provide a useful constraint on the vertical distribution of heterogeneity within the mantle. There are two reasons for this. While the onset of scattered energy depends strongly on the vertical distribution of heterogeneity, the coda decay rates are nearly insensitive to this. The second reason is that the statistical variability of coda amplitudes is high. The standard errors of the stacked data are greater than the difference between the coda amplitudes predicted by the two models.

Our analysis of simulated and recorded data indicates that $P K P$ precursors remain the most effective probe of small-scale heterogeneity in the mantle. Specifically, the increase in the amplitude of the precursors with time and increasing range appears to be a diagnostic indicator of the vertical distribution of the heterogeneity. Our analysis of 3624 high-quality teleseismic recordings confirms the conclusions of Hedlin et al. (1997). PKP precursors are consistent with scattering uniformly distributed throughout the lower mantle and not with scattering confined to the CMB. The preferred model requires $\sim 1 \%$ RMS heterogeneity with a scale length of $8 \mathrm{~km}$ at all depths..

\section{ACKNOWLEDGEMENTS}

This research was made possible by high-quality data in the FARM archive at the IRIS DMC. This research was funded by National Science Foundation EAR99-093370.

\section{REFERENCES}

Chernov, L.A., Wave propagation in a random medium (trans. by Silverman, R.A.), McGraw-Hill, New York, 1960.

Doornbos, D.J., and E.S. Husebye, Array analysis of PKP phases and their precursors, Phys. Earth Planet. Int., 5, 387, 1972.

Efron, B., and R. Tibshirani, Statistical data analysis in the computer age, Science, 253, 390-395, 1991.

Haddon, R.A.W., Corrugations on the CMB or transition layers between inner and outer cores?, Trans. Am. Geophys. Union, 53, 600, 1972.

Haddon, R.A.W., and J.R. Cleary, Evidence for scattering of seismic PKP waves near the mantle-core boundary, Physics of the Earth and Planetary Interiors, 8, 211-234, 1974.

Hedlin, M.A.H., P.M. Shearer, and P.S. Earle, Seismic evidence for small-scale heterogeneity throughout the Earth's mantle, Nature, 387, 145-150, 1997.

Hedlin, M.A.H. and P.M. Shearer, 2000, An analysis of large-scale variations in small-scale mantle heterogeneity using Global Seismographic Network recordings of precursors to PKP, Journal of Geophysical Research, 105, 13655-13673.

Husebye, E.S., D.W. King, and R.A.W. Haddon, Precursors to PKIKP and seismic wave scattering near the mantle-core boundary, Journal of Geophysical Research, 81, 1,870-1,882, 1976.

Pechmann, J.C., S. J. Nava, J.C. Bernier and W.J. Arabasz, 2000, A Critical Analysis of Systematic Time-Dependent CodaMagnitude Errors in the University of Utah Earthquake Catalog, 1981-1999, EOS, 81, F869. 